\title{
Schmerztherapie muskuloskelettaler Krankheiten
}

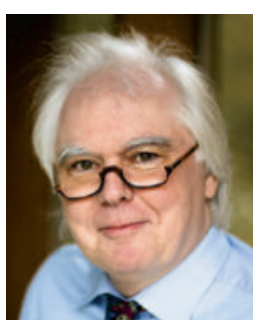

Prof. Dr. Wolfgang Rüther

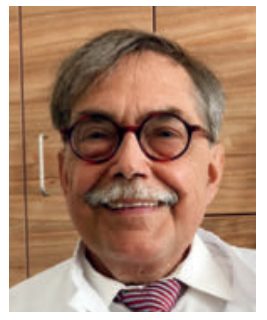

Prof. Dr. Jörg Jerosch
Liebe Kolleginnen, liebe Kollegen,

die neue Weiterbildungsordnung wird in diesem Jahr sukzessive von den Landesärztekammern verabschiedet und in Kraft gesetzt werden. Für die Zusatzweiterbildung Orthopädische Rheumatologie bringt sie umfassende Änderungen. Waren bisher die Erkennung und die operative Therapie die zentralen Ziele dieser Zusatzweiterbildung, nehmen jetzt die Diagnostik und die konservative Therapie der verschiedenen Formen rheumatischer Krankheiten den größeren Raum ein. Die großen Subdisziplinen konservativer muskuloskelettaler Medizin sind in der neuen Weiterbildungsordnung dezidiert vertreten: Schmerztherapie, manuelle Medizin, Osteologie, Rehabilitation, physikalische Therapie, technische Orthopädie, medikamentöse Therapie und - natürlich - die operative Rheumatologie.

Bei der Umsetzung der Weiterbildung Orthopädische Rheumatologie werden in Zukunft außer der DGORh weitere Fachgesellschaften innerhalb der Deutschen Gesellschaft für Orthopädie und Unfallchirurgie DGOU zu beteiligen sein, die sich den o. g. Subdisziplinen klinisch und wissenschaftlich widmen. Sie verfügen neben einer großen Zahl von Fachleuten teils über ein breites und bewährtes Kursprogramm, das während der Weiterbildung zum Kenntniserwerb und zur -vertiefung genutzt werden kann.

Das vorliegende Heft thematisiert schmerztherapeutische Aspekte. Federführend beteiligt ist die Interdisziplinäre Gesellschaft für orthopädische/unfallchirurgische und allgemeine Schmerztherapie IGOST. Diese wissenschaftliche Gesellschaft vertritt innerhalb der DGOU das Thema Schmerz. Prof. Jerosch ist als Vorstandsmitglied der IGOST Mitherausgeber dieses Heftes.

Die angesprochenen Themen sind speziell. Sie führen uns in Bereiche der Schmerztherapie, die nicht alltäglich sind, unseres Erachtens aber den Horizont für neue Gedanken öffnen: Injektionen an der Wirbelsäule mittels Sonografie, Embolisation synovialer Gefäße bei Gonarthrose, Bedeutung des Schmerzes bei der Begutachtung. Die Beiträge zur Strahlentherapie gutartiger muskuloskelettaler Krankheiten und zur perioperativen Schmerztherapie liefern interessante Updates. Schließlich eröffnet uns Raimund Casser, langjähriger Vorstand der IGOST, seine Einschätzung zu den Zukunftsperspektiven: Zu Recht fordert er die spezielle orthopädische Expertise in der Schmerztherapie muskuloskelettaler Krankheiten - auf dem Weg zur „Speziellen orthopädischen Schmerztherapie“?

In folgenden Ausgaben der arthritis + rheuma wollen wir in loser Reihenfolge die o. g. Subdisziplinen konservativer muskuloskelettaler Medizin zu Wort kommen lassen und dadurch die jetzt entfaltete Breite der Orthopädischen Rheumatologie darstellen.

Wir wünschen Ihnen eine anregende Lektüre.

Ihre

Prof. Dr. Wolfgang Rüther

Prof. Dr. Jörg Jerosch 